\title{
TIPOLOGÍA DE LAS ORGANIZACIONES DE LA REGIÓN DE CUAUHTÉMOC, CHIHUAHUA
}

\author{
Ema Cristina Gutiérrez Enríquez*, Karla Isabel Gutiérrez Enríquez ${ }^{* *}$, Paola Molinar Merino**, \\ Sonia Pérez Gómez**
}

Gutiérrez-Enriquez E.C., Gutiérrez-Enriquez K.I., Molinar-Merino P., Pérez-Gómez S.,Tipología de las Organizaciones de la región de Cuauhtémoc, Chihuahua. Hitos de Ciencias Económico Administrativas 2016;22 (62): 7-16

\section{RESUMEN}

Objetivo: Describir la tipología de las organizaciones de la región de Cuauhtémoc.

Materiales y método: Se visitaron 35 organizaciones que tuvieran más de 15 empleados y más de 15 años de vida, se realizó una entrevista dirigida con los empresarios.

Resultados: Se encontró que la mayoría de las empresas visitadas son pequeñas empresas y cuentan con una antigüedad de veinte a cincuenta años, en un $60 \%$ de ellas aun labora el fundador.

Conclusiones: Se concluye que las empresas visitadas se encuentran laborando bajo un esquema de «empresas familiares».
Gutiérrez-Enriquez E.C., Gutiérrez-Enriquez K.I., Molinar-Merino P., Pérez-Gómez S.,Tipología de las Organizaciones de la región de Cuauhtémoc, Chihuahua. Hitos de Ciencias Económico Administrativas 2016;22 (62): 7-16

\section{ABSTRACT}

Objective: Describe the types of organizations in the region Cuauhtémoc.

Material and method: 35 organizations that have more than 15 employees and more than 15 years of life he visited an interview conducted with entrepreneurs was held.

Results: It was found that most of the companies visited are small and have a length of twenty to fifty years, $60 \%$ of them still works the founder.

Conclusions: It is concluded that the companies visited are laboring under a scheme of «family businesses».
Palabras clave: Tipología empresarial. Clasificación de las empresas.
Keywords: Business typology. Classification of companies.

DIRECCIÓN PARARECIBIR CORRESPONDENCIA: Correo electrónico: cristigutie@gmail.com

\footnotetext{
* Maestra en C. Instituto Tecnológico de Cd. Cuauhtémoc. Departamento de Ciencias Económico - Administrativas.

Cuauhtémoc, Chihuahua.

** Ingeniera en C. Instituto Tecnológico de Chihuahua. Departamento de Ingeniería industrial. Chihuahua, Chih
} 
$\mathbf{L}$

as organizaciones nacieron con el fin de satisfacer las necesidades de los individuos, por lo que se puede remontar sus orígenes desde el inicio de la sociedad, cuando el hombre era nómada, requería del apoyo de un grupo de personas para poder realizar su actividad principal, la caza, así en el momento de permanecer a dicho grupo estaba formando parte de una organización y al mismo tiempo estaba satisfaciendo una de las necesidades básicas de todo ser humano, la alimentación y el vestido. Con el tiempo y con el sedentarismo del hombre esto fue siendo un poco más formal, se satisfacían las necesidades mediante el intercambio de mercancías (trueque) de persona a persona, familias a familias; formando organizaciones que se regían bajo las normas establecidas por los involucrados en dicho negocio.

Rodríguez (1992), menciona que en México la industrialización se inició de manera firme y continuada en la década de los 50, en la que surgió la actividad industrial con un sinnúmero de empresas pequeñas y medianas, gracias a la acción abierta y decidida del Gobierno Mexicano, no solamente a través de la integración de una infraestructura básica, sino también promoviendo inversiones industriales que permitieron disminuir las importaciones de artículos manufacturados y para mantener un nivel de empleo adecuado a las necesidades de una población cada vez más crecientes.

En 1955 se promulgó la «Ley para el Fomento de las Industrias Nuevas y Necesarias", cuyo ordenamiento permitió que se estableciera un importante número de empresas industriales, fundamentalmente medianas, pequeñas, y que un gran número de talleres y artesanías se transformaran en pequeñas empresas.

Históricamente, la empresa pequeña y mediana ha ofrecido una alternativa para el proceso de industrialización y que ofrecen una oportunidad para una continuada expansión del sistema económico del país. Las pequeñas y medianas empresas existen en tres ramas principales, estas son: la industrial, la comercial y de servicios. Es indiscutible que las empresas prosperen gracias a la aplicación de modernas técnicas de administración. Lo que importa realmente en la empresa es la iniciativa de su jefe, su capacidad de hacer frente a los problemas que plantea la dirección; su éxito depende del conocimiento, y aplicación de la administración.

El ambiente de incertidumbre en los sectores políticoeconómico y sobre todo en el social, ha obligado a las organizaciones a modificar su formas de trabajo, en cuanto a sus grupos, procesos, manera de pensar y actuar, buscando establecer equipos de trabajo autodirigidos que sean autosuficientes y productivos, para ello, es necesario que las personas dentro de cada organización cambien su pensamiento rígido y se capaciten, de tal forma que lleguen a tener el poder suficiente de tomar decisiones, establecer objetivos, automotivarse, planear y evaluar su propia actividad hacia el logro de los objetivos organizacionales.

Lo antes expuesto, toma mayor relevancia cuando los miembros de la organización pertenecen a distintas culturas e idiosincrasias, problemática que se presenta en la región de Cuauhtémoc, Chihuahua, dado que la composición cultural de las empresas, principales y emergentes, es propiedad de la comunidad menonita y con trabajadores mestizos provenientes de las comunidades urbanas y rurales de la zona y de otras regiones del país. Dada esta estructura multicultural, el liderazgo, la estructura de grupo, la capacidad innovativa y la resistencia al cambio por parte de las organizaciones y sus integrantes, están en función de las dimensiones culturales que presentan cada uno de los grupos étnicos que interactúan entre sí, lo cual genera diferencia en la visión de los objetivos y métodos para llegar a ellos, teniendo divergencias en cuanto al logro de los resultados y provocando un deficiente desarrollo organizacional.

Por lo cual, en la presente investigación se realiza un análisis de la tipología de las organizaciones de la región de ciudad Cuauhtémoc, en la cual participaron 35 empresarios de la misma región, proporcionando datos relevantes de sus respectivas empresas, que ayudaron a realizar una clasificación y análisis de éstas. 


\section{MARCO TEÓRICO CONCEPTUAL}

La historia empresarial de ciudad Cuauhtémoc, se ha dado en un proceso similar a la evolución de las organizaciones en el mundo, a este respecto, autores como Martínez (2008), describe que es en el año de 1869, en el mes de febrero, cuando se hace la separación territorial de San Antonio de los Nogales, San Antonio de Loya y San Antonio de Arenales en la municipalidad de Cusihuiriachic, quedando en el año de 1880 el territorio de San Antonio de los Arenales como un rancho de la hacienda de Bustillos. Esta asignación territorial, da lugar a la creación de una estación ferroviaria justamente en la sección de San Antonio de los Arenales, la cual fue inaugurada el 31 de Diciembre de 1899, lo que da pie a la formación de una nueva población, pues ya que para el año de 1910, San Antonio de los Arenales tiene una población de 173 habitantes y junto a la estación de ferrocarril se encontraba una bodega para almacenar granos, perteneciente a la Familia Zuloaga.

El autor anterior comenta que el 8 de Marzo de 1922, llega a la región, un grupo de menonitas procedentes de Canadá, los cuales emprenden la valiosa labor de trabajar el campo y la producción de quesos. Desde sus inicios, los primeros pobladores de la región de Cuauhtémoc, tanto menonitas, como los mestizos, mostraron tener una gran calidad humana, inteligencia, capacidad de trabajar, entusiasmo, ganas de salir adelante, ya que la necesidad de sacar adelante a sus familias, los llevó a desarrollar una habilidad empresarial, puesto que empezaron a trabajar la tierra y a comercializar sus productos, lo cual dio origen al surgimiento de empresas de comercialización y posteriormente de industrialización de los productos del campo, como semillas, fruta y derivados de la leche.

Los hechos históricos, dan una idea de lo que la población de Cuauhtémoc ha desarrollado en los ámbitos social y económico, de tal forma que el crecimiento poblacional, muestra un cuantioso aumento en número de pobladores y de empresas, donde se conjugan dos grupos sociales y dos culturas, dando lugar a un fuerte vínculo empresarial, según datos de la Presidencia Municipal de Cuauhtémoc (2008).
El municipio de Cuauhtémoc, a través de Fomento Económico (2008), tiene un registro de 3,950 empresas, algunas de ellas con una larga trayectoria empresarial; lo que da pie para considerar que en la región de Cuauhtémoc, existen empresas y empresarios exitosos, que mediante su forma, técnica y estrategias utilizadas para dirigir, les ha permitido mantenerse al frente de la organización, y han sobrevivido en el mercado.

Existen diferentes maneras de definir las organizaciones, muchas veces su concepto depende del tema del que se esté hablando, del enfoque que se le quiera dar, o de lo que se quiera resaltar. Münch (2009), hace mención de algunos autores que las definen con un enfoque administrativo: Antony Jay (1971), las señala como instituciones para el empleo eficaz de los recursos para mantener y aumentar la riqueza de los accionistas y proporcionarles seguridad y prosperidad a los empleados.

El Diccionario de la Real Academia Española (1999), las define como una entidad integrada por el capital y el trabajo, de los factores de producción y dedicada a actividades industriales, mercantiles o de prestación de servicios, con fines lucrativos y la consiguiente responsabilidad. José Antonio Fernández Arena (1973), comenta que son una unidad productiva o de servicio que, constituida según aspectos prácticos o legales, se integra por recursos y se vale de la administración para lograr sus objetivos; Petersen y Plowman (2009), puntualizan que es una actividad en la cual varias personas cambian algo de valor para obtener una ganancia o utilidades mutuas.

Mientras que Rodriguez (2002), añade que desde el punto de vista económico, es una entidad económica destinada a producir bienes y servicios, venderlos, satisfacer el mercado y obtener un beneficio. $Y$ hace referencia de las definiciones de: Guzmán Valdivia (1980), que las define como la unidad económico-social en la que el capital, el trabajo y dirección se coordinan para lograr una producción que responda a los requerimientos del medio humano en que la propia empresa actúa y de Roland Caude (2002), que menciona que son un conjunto de actividades humanas colectivas 
organizadas con el fin de producir bienes o rendir beneficios.

Hall (1983), menciona que la tipología más común es la que Warriner (1980), ha denominado tradicional, folclórica o de sentido común, de tal manera las organizaciones pueden clasificarse en dos categorías: con ánimo de lucro o sin ánimo del mismo. El aspecto importante no es debatir la importancia de tener o no una orientación hacia el lucro. Es indudable que tener una orientación hacia el lucro, o empresarial podría ser consideración importante en un esfuerzo tipológico serio, si se recuerda que muchas organizaciones públicas se embarcan en esfuerzos empresariales al buscar fondos y muchas otras que se consideran con ánimo de lucro están, en industrias reguladas, tan protegidas de la competencia que la obtención de lucro difícilmente aparecería como un aspecto de mucha importancia.

Otra forma de tipología, de sentido común, sería clasificar las organizaciones por sector social, tales como: el educativo, el agrícola, el de salud y médico, entre otros.

Prieto (1994), cree que una forma muy singular y acertada para saber si una empresa es chica, mediana o grande, es la que determinan los expertos consultores nacionales y privados. Por ejemplo, en nuestro país, Nacional Financiera, institución de crédito del Gobierno Federal, hace una clasificación a este apartado, señalando que una empresa grande es aquella que vende más de 2,010 veces el salario mínimo general elevado al año y tiene más de 250 empelados; una empresa mediana es aquella que vende hasta 1,115 veces el mismo salario elevado al año y tiene más de 100 empleados, quedando para la empresa pequeña los rangos inferiores.

Como es posible darse cuenta, el volumen de las ventas y el número de personal ocupado en una empresa, pueden ser dos factores, o dos elementos de juicio razonable para poder dividir a las empresas en grandes, medianas y pequeñas. Cabe señalar, que la microempresa es aquella que tiene hasta 15 empleados y sus ventas son menores a 110 veces el salario mínimo general elevado al año.

Como se observa según los diferentes autores, son diversas las clasificaciones que se presentan para las organizaciones, Münch (2009), las clasifica por actividad o giro, como industriales, las cuales pueden ser extractivas o manufactureras, las comerciales que se dividen en mayoristas, minoristas y de comisión, y por último las de servicios públicas o privadas. El tamaño de las empresas se determina de diferentes formas, por monto de capital, por número de personas que laboran en ella, por nivel de tecnificación y el nivel de impacto en las ventas.

La magnitud de la empresa proporciona la siguiente información en ese sentido, el 30 de marzo de 1999 la Secretaría de Comercio y Fomento Industrial, (SECOFI), resaltó que los nuevos criterios a considerar toman en cuenta el número de empleados de cada establecimiento como única variable de estratificación, como criterios complementarios se consideran el total de ventas netas anuales y de activos fijos.

Pinilla y compañía (1994), clasifican la empresa según el número de propietarios, como individual con un solo dueño y societaria cuando hay más de dos propietarios Según Cano (2006), el cambio organizacional se puede entender "como una modificación del estado de cosas en algo que nos atañe o importa; o una transición en nuestro status quo, para mejorar o empeorar». El autor cita a Kauffman, (1997), quien argumenta que el proceso de cambio «es el medio de que dispone una organización para transformarse y aplicar una nueva visión». Así mismo, el autor menciona que si las organizaciones están interesadas en mantenerse en un mercado competitivo, deberán adaptarse rápidamente a las demandas del entorno económico, político y social. Señalando que el entorno incluye clientes, proveedores, comunidad, y que debe abarcarlos todos para asegurar el éxito. Los cambios drásticos tecnológicos obligan a las organizaciones a cambiar sus perspectivas y estrategias empresariales. 
El autor argumenta que «los países, y sobre todo los subdesarrollados deben enfrentar la radical transformación que se está produciendo en las condiciones de competitividad y de inserción en la economía mundial, desarrollando organizaciones y programas que impliquen nuevas estrategias de mercado». Por tanto, «el cambio debe darse en la cultura empresarial, en donde las condiciones de los mercados son las que marcan la pauta para incorporar nuevas tendencias en la dirección y administración de las organizaciones, tarea que de no hacerse, implica una gran probabilidad de desaparecer». Todas las organizaciones privadas y públicas están obligadas a cambiar permanentemente sus estructuras, objetivos y estrategias de tal forma que puedan estar preparadas para enfrentar las nuevas exigencias de la globalización, llegando a ser de clase mundial.

Las organizaciones de clase mundial se enfocan a la satisfacción del cliente a través de la calidad en los productos, bajo costo y competitividad, sus características principales son: la capacitación; la búsqueda de la mejora continua; el desarrollo de ventajas competitivas y un sistema de negocios integral, tienen una visión del futuro; son sensibles a las necesidades cambiantes del mercado; enfatizan el conocimiento profundo de sus procesos de trabajo; se apegan y promueven normas y estándares mundiales, en si promueven la mejora continua. El contar con todo lo anterior les da una certificación y un reconocimiento mundial. Cano (2006), concluye que «el cambio es la única constante y trae consigo nuevas formas de ver y concebir las cosas.

Existen tendencias derivadas de los mercados globalizados que promueven el establecimiento de normas y leyes que sirven como punto de referencia para que las empresas se transformen. Por tanto, la dirección de las empresas de clase mundial requiere de líderes que no sólo visualicen y afronten con éxito estos cambios, sino que los provoquen. Ante esto, la sobrevivencia, crecimiento y competencia de las nuevas organizaciones depende en gran medida de un liderazgo creativo y desarrollador que motive al recurso humano a ser productivo y lograr los objetivos institucionales».
Estas nuevas tendencias organizacionales de humanizar los procesos y aplicar el comportamiento organizacional en la mayoría de las acciones dentro de la organización, han conducido a la exigencia de personal más capacitado, con mayor compromiso con las actividades diarias, con las acciones del equipo de trabajo y con los objetivos de la organización. Parte de ello, es derivado precisamente de las tendencias de globalización que se tienen en este contexto. De ahí que la globalización afecte las tendencias y decisiones en el plano del comportamiento organizacional.

La globalización ha eliminado de cierta forma las fronteras geográficas entre los países, Arámbula (2003), comenta que "la globalización tiene un impacto determinante en la sociedad, en su cultura, en su modo de vida, su forma de hacer negocios, a tal grado que la misma ha tenido apoyo y rechazo de gran parte de la humanidad. Quienes la aceptan ven oportunidades de negocio, de expansión, dominio, crecimiento y riqueza. Quienes la rechazan ven pérdida de valores, de cultura, de identidad nacional, y la amenaza de competir en desventaja con las grandes potencias». Esta situación obliga a las organizaciones e individuos a cambiar y adaptarse rápidamente ante estas exigencias sociales y económicas, si no lo hacen se quedan en el camino.

Las organizaciones tienden a hacerse esbeltas en sus procesos, rápidas y enfocadas a clientes y productos, así como innovadoras, con la idea de subsistir en el Siglo XXI.

La globalización no tiene vuelta atrás y el éxito de las empresas y organizaciones dependerá de su capacidad de adaptarse al cambio, de innovarse y procesar la información. El autor argumenta en relación a la conservación de la cultura en cada país y región frente la influencia de un mundo globalizado, que cada uno de ellos decidirá si mantiene sus raíces o se globaliza. Ante esto las compañías «se están preparando para enfrentar la globalización con éxito; una de las estrategias adoptadas es la de fortalecer la cultura organizacional. En estas empresas, la cultura regula el comportamiento hacia el éxito". 
Para Arámbula (2003), la aplicación del comportamiento organizacional al manejo y entendimiento del personal en la organización, son determinantes en el poder adaptarse al cambio y a la globalización, ya que solo se logrará mediante la aceptación y participación del empleados. Esto implica un gran reto organizacional, señala Mora (2008), ya que las organizaciones deben adaptarse a personas que son diferentes entre sí, creando una diversidad laboral, lo cual ha hecho a las organizaciones heterogéneas al contener entre su personal la procedencia de diferentes razas, lugares de origen, ideologías, creencias, culturas; obligando a la organización a entenderlos y guiarlos hacia el objetivo principal, sin perder de vista el bienestar del personal. Los administradores tienen que cambiar su filosofía de tratar a todos por igual y reconocer las diferentes capacidades de las personas. El autor comenta, que las organizaciones ya no se ven afectadas por las fronteras geográficas de cada país, sino que la globalización convirtió al mundo en un solo lugar, por ello los administradores deben ser capaces de trabajar con personas de diferentes culturas.

Así mismo, el administrador está cada vez más expuesto a realizar trabajos en el extranjero o con personas extranjeras, para lo cual deberá de comprender sus culturas y adaptarse a esas diferencias. El autor argumenta que «las organizaciones exitosas deben fomentar la innovación y dominar el arte del cambio o se pondrán en peligro de extinción. El éxito lo alcanzarán las organizaciones que mantengan su flexibilidad, mejoren constantemente la calidad y venzan a la competencia del mercado con una corriente continua de productos y servicios innovadores».

\section{METODOLOGÍA}

El proyecto se realizó en la región de ciudad Cuauhtémoc, considerando para análisis, empresas que tengan más de 15 empleados y más de 15 años de existencia. Se aplicaron las encuestas con el propietario de la organización o el gerente de la misma.

El objetivo de investigación, es el de describir la tipología de las organizaciones de la región de Cuauhtémoc. De esta forma, se obtendrán las características principales que predominan en esta región.
1. El Instrumento de medición que se utilizo fue, una encuesta diseñada por Gutiérrez 2010, con 34 preguntas cerradas y abiertas de respuesta libre. Para ello se visitó personalmente a cada una de los empresarios, contestando la encuesta mediante una entrevista.

2. La muestra de empresas se llevó a cabo por cuota, y a conveniencia, considerando 35 organizaciones que cumplían con las características requeridas. Para ello se visitó al propietario o al gerente de la organización, se expuso ante ellos el objetivo a lograr con el estudio y se contó con su consentimiento para proporcionar la información requerida y utilizarla para el análisis. Para la selección de las empresas, se utilizó el padrón de registro de empresas de Fomento Económico del municipio.

3. El análisis de los datos se llevó a cabo mediante procedimientos estadísticos descriptivos, a través de los cuales se obtuvo el porcentaje de empresas que cumple con la característica esperada.

\section{RESULTADOS}

Se visitaron 35 empresas para recabar datos organizacionales que definieran las características de su estructura, tamaño, giro, la información obtenida fue la siguiente y se observa en la figura siguiente:

La mayoría de las empresas encuestadas, se encontró que fueron fundadas entre 1960 y 1980.

El promedio de edad de los empresarios es de 52 años, en su mayoría casados y una escolaridad de promedio bachillerato. De las personas encuestadas 25 son del sexo genérico masculino, 10 del sexo femenino.

El $57 \%$ de los empresarios que fueron encuestados son los fundadores de la empresa, y aún están en activo.

El $77 \%$ de los empresarios expresan que la familia se involucra en la organización, en las áreas de administración, en ventas, en compras, como cajeros, secretarias (os), en oficina, en capacitación del personal, en laboratorio, como gerentes, en eventos sociales, en taller, en laboratorio, en todas las actividades que se realizan en la empresa, entre otras. 
El $91 \%$ de las empresas visitadas son pequeñas. El $63 \%$ de las empresas visitadas es del giro comercial y el $37 \%$ restante son de servicios.

Referente a la estructura organizacional de las empresas, el $48 \%$ de ellas cuentan con una estructura formal y $52 \%$ restante funciona con una estructura informal.

En el $71 \%$ de las empresas se cuenta con los servicios de al menos un profesionista.

La materia prima para la producción o los productos que comercializan las empresas se obtiene en el $60 \%$ de los casos, de empresas de origen nacional, el 14\% es de origen extranjero y el $20 \%$ de ambos lugares, nacional y extranjero.

El área de influencia de la empresa fue que el 57\% de las empresas tienen un rango de influencia regional, el $28 \%$ de influencia local y el $14 \%$ son de influencia nacional.

Las estrategias que los empresarios utilizan para salir adelante en tiempos o épocas difíciles económicas o socialmente son: primeramente se realiza un análisis de la situación actual, acto seguido se revisan los registros anteriores y en base a esto aunado a la experiencia se toman decisiones.

En el $49 \%$ de las empresas realizan cambios de manera no muy frecuente, una vez cada dos años y 1 el $51 \%$ de las empresas no realizan cambios.

El 94\% de los empresarios permiten que los empleados participen en el establecimiento de metas, objetivos, procesos, cambios.

El 94\% de los empresarios promueve el trabajo en equipo.

El 25\% de los empresarios cuenta con socios. En las 35 empresas encuestadas se brinda trabajo a un total de 477 personas (ver figura 1).

\section{CONCLUSIONES}

Se tenía previsto entrevistar a 52 empresarios, solo se logró entrevistar a 35 de ellos que accedieron a proporcionar la información, de los 17 restantes, 7 dijeron que no deseaban contestar la encuesta por motivos de seguridad personal, para no meterse en problemas o porque sencillamente no les interesaba, los otros 10 no se lograron encontrar en las varias ocasiones que se visitaron las instalaciones de las empresas contempladas, por lo que se decidió después de varios intentos de contactarlos, incluirlos en la parte de no entrevistados.

La mayoría de las empresas encuestadas y visitadas para fines de este estudio, son de tipo familiar y son consideradas como pequeñas ya que cuentan con menos de 20 empleados y se encuentran en el giro de comercio o servicios, sin embargo dan trabajo a 477 empleados.

Se observa, que la edad promedio de los empresarios es de 52 años, lo cual indica que iniciaron en el ramo empresarial a edad temprana, acumulando años de experiencia a este momento. Otro aspecto que resalta es que la mayoría de las empresas están dirigidas por hombres, coincidiendo con la tendencia social de predominar el hombre como el fuerte del grupo.

Gran parte de los empresarios promueven la participación de los empleados en el establecimiento de objetivos y metas, así como fomentan el trabajo en equipo, lo cual se considera con una tendencia moderna de trabajo organizacional.

El $50 \%$ de las empresas aún no cuenta con una estructura formal y se detectó que son pocos los cambios que se realizan en la empresa y no muy frecuentes, por lo que permanecen estables, aspecto que se requiere para mantenerse a la vanguardia; sin embargo, una de las fortalezas observadas, es que realizan análisis de la situación de mercado y de los datos financieros lo cual consideran importante.

A pesar de ser organizaciones pequeñas y familiares, la mayoría de las empresas encuestadas, cuenta con el apoyo de profesionistas entre su personal laborando. 


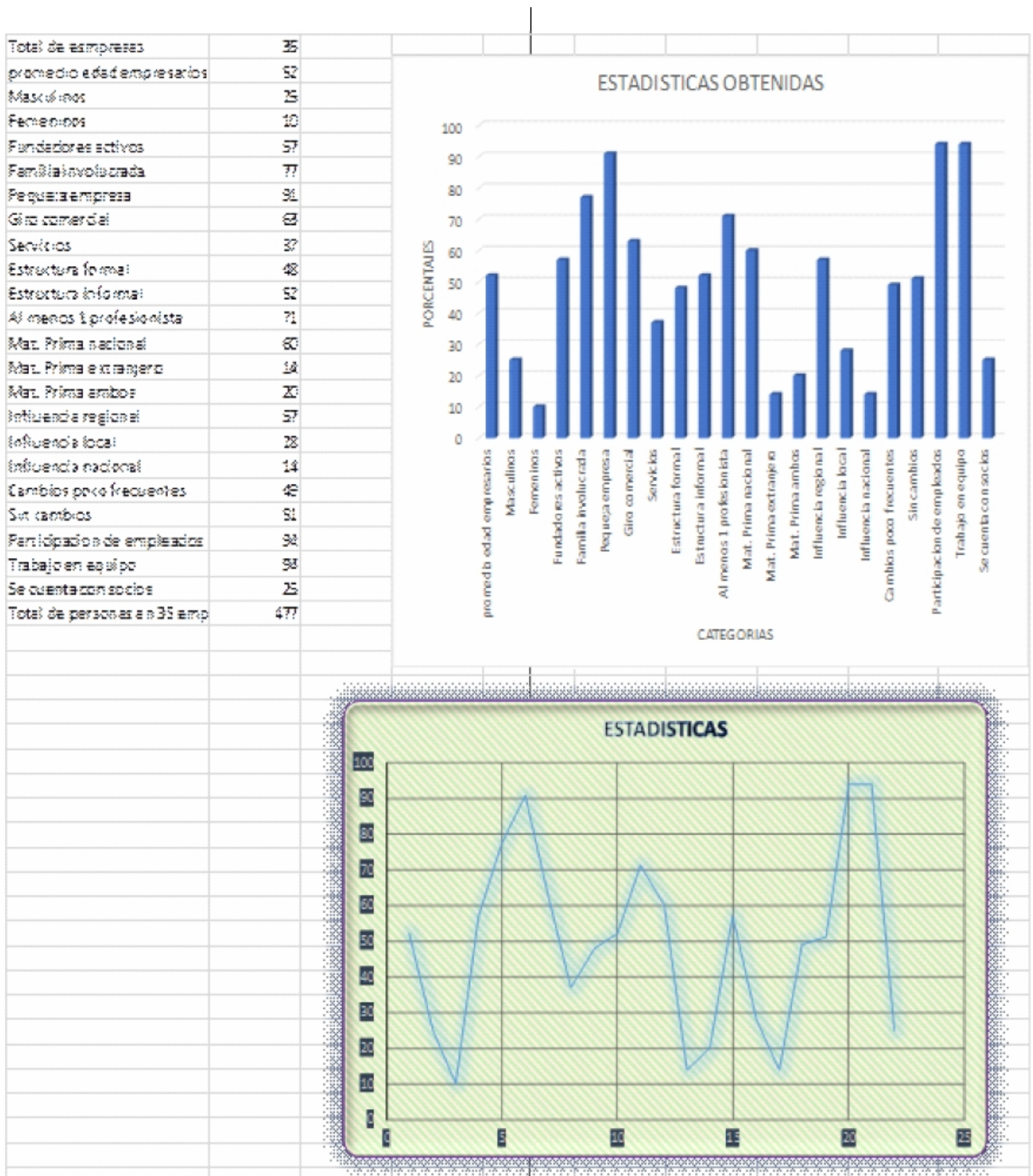

Figura 1. Porcentaje de las Características Empresariales.

Fuente: Elaboración propia.

Los consejos que los empresarios dieron a los jóvenes emprendedores son: ser creativos, buscar la forma de crear algo nuevo para la comunidad, echarle ganas, el que no arriesga no gana, siempre estar actualizándose sobre los cambios que día a día existen, buscar y encontrar estrategias para mejores resultados, tener responsabilidad en lo que emprendan, tener mucha visión, con empeño y dedicación se pueden lograr muchas metas, implementar las nuevas herramientas adquiridas en sus estudios sin dejar a un lado la experiencia de quienes están ya en el campo de trabajo, que se capaciten, hay estrategias de atención al cliente, mercadotecnia y financieras.

Primeramente, hacer un estudio antes de iniciar una empresa para evitar fallos, proyectar algo pero 
analizarlo a fondo, tener ganas de capacitarse y trabajar duro, ser responsables y emprendedores para un mejor futuro, la superación por medio del estudio, ponerle ganas y empeño al trabajo del negocio, crear ideas nuevas, trabajar mucho y capacitarse, trabajar duro para salir adelante, trabajar 12 horas diarias, 360 días del año, no caer en la rutina y no desmayar, ser constantes, no perder la fe, tener iniciativa, tener actitud de servicio, cortesía para los clientes y compañeros, ser disciplinados, observar necesidades y demandas, realizar lo que les guste, luchar por lo que quieren, perseverar, estar al pendiente de lo que hacen los demás, trabajar en equipo, dar motivación a los empleados, emprender acciones innovadoras y productivas, prepararse, actualizarse permanentemente, tener expectativas del mercado, investigar el negocio en el que se desea iniciar, ser honestos, prepararse para la globalización y cumplir todas sus metas.

\section{REFERENCIAS}

Arámbula, M. (2003). El impacto de la globalización en la formación de una cultura organizacional. Recuperado de http://207.249.140.110/cgi-bin/ contenido.pl?Art=286

Cano, M. (2006). El liderazgo transformacional: sustento de las organizaciones de clase mundial. Instituto de Investigaciones y Estudios Superiores de las Ciencias Administrativas. México: Universidad Veracruzana. Recuperado de http://www.uv.mx/iiesca/revista/documents/ liderazgo2006-2.pdf

Fernández, J. (1973). Introducción a la administración. (Cap. 3-4). México: UNAM.

Hall, R. (1983). Organizaciones, estructura y proceso. ( $3^{r a}$ ed.). México: Prentice Hall hispanoamericana.

Real Academia Española, diccionario de la lengua Española, 1999, (2ª ed.). Barcelona.

Martínez, M. (2008). Cronología e historia de Cuauhtémoc. Chihuahua. México: Presidencia Municipal de Cuauhtémoc. Recuperado de www.cuauhtemoc.gob.mx

Mora, C. (2005). Organizaciones exitosas del siglo XXI. Venezuela: Área de estudios de Postgrado de la Universidad de Carabobo. Recuperado de www.cmoraarrobapostgrado.uc.edu.ve y www. camv12arrobahotmail.com

Münch, G. (2009). Fundamentos de administración. (8 $8^{\text {a }}$ ed.). México: Editorial Trillas.

Presidencia Municipal de Cuauhtémoc. (2008). Conoce más de Cuauhtémoc. Características geográficas. Recuperado de www.cuauhtémoc.gob.mx

Presidencia Municipal de Cuauhtémoc. (2008). Fomento Económico. Chihuahua, México: Base de datos de las Empresas del Municipio.

Pinilla, F., Lázaro M., y Martínez J. (1994). Procesos y fundamentos de administración y gestión. México: Mc Graw Hill.

Prieto, C., y Suárez C. (1994). Introducción a los negocios, ( $3^{\underline{a}}$ ed.). Primera parte: Operación de la Empresa. México: Editorial banca y comercio.

Rodríguez, J. (2002). Organizaciones contables y administrativas de las Empresas. (3ra ed.). México: Thomson.

Rodríguez, J. (1992). Como administrar pequeñas y medianas empresas. México: Editorial ECASA

Secretaría de Comercio y Fomento Industrial. (1999). Estratificación Empresarial. México: Diario Oficial de la Federación. 
